\title{
Evaluation of Two Noise Level Prediction Models: Multiple Linear Regression and a Hybrid Approach
}

\author{
Peter Ekow Baffoe ${ }^{1, *}$, Alfred Allen Duker ${ }^{2}$ \\ ${ }^{1}$ Department of Geomatic Engineering, Faculty of Mineral Resources Technology, University of Mines and Technology, Tarkwa, Ghana \\ ${ }^{2}$ Department of Geomatic Engineering, School of Engineering, Kwame Nkrumah University of Science and Technology, Kumasi, Ghana
}

\section{Email address:}

pebaffoe@umat.edu.gh (P. E. Baffoe), duker@alumni.itc.nl (A. A. Duker)

${ }^{*}$ Corresponding author

\section{To cite this article:}

Peter Ekow Baffoe, Alfred Allen Duker. Evaluation of Two Noise Level Prediction Models: Multiple Linear Regression and a Hybrid Approach. American Journal of Mathematical and Computer Modelling. Vol. 4, No. 3, 2019, pp. 91-99. doi: 10.11648/j.urp.20190403.12

Received: May 9, 2019; Accepted: June 17, 2019; Published: July 26, 2019

\begin{abstract}
Noise prediction models are very useful for urban planning and environmental management. As a result researchers are always searching for methods that are practically applicable in predicting noise levels accurately. It therefore became paramount to implement special systems that could to predict noise levels accurately for an urban area. In this study two land-use regression methods, were used to formulate two noise level prediction models namely, multiple linear regression (MLR) and analytical hierarchy process (AHP)-multiple linear regression for the Tarkwa Mining Community (TMC). The performances of the two models were evaluated using statistical indicators. The MLR model performed better than that of a hybrid model of AHP-MLR with RMSE of 1.569 , standard deviation of $1.585, \mathrm{R}^{2}$ of 0.961 and $\mathrm{R}$ of 0.980 . The performance of the hybrid AHP-MLR was also RMSE of 1.774 , standard deviation of $1.758, \mathrm{R}^{2}$ of 0.955 and $\mathrm{R}$ of 0.977 . Plotted box-andwhisker and range plots further confirmed the performances of the two models. The resulting map from the noise prediction gave insight suggested that with the appropriate data and useful tools noise pollution levels of an urban area could be well predicted and mapped for urban planning and environmental management.
\end{abstract}

Keywords: Noise Level, Noise Pollution, Noise Models, Land Use Regression Models

\section{Introduction}

The levels of environmental noise pollution are increasing at an alarming rate in our urban communities, especially the mining communities. This is primary due to infrastructural development, industrial activities, social activities and high influx of people from different cultural backgrounds, with implication of increased generation of noise. Studies have confirmed in literature that increase in noise pollution brings about increase in risk factor for cardiovascular dysfunction, ear problems including hearing impairment, sleep disturbances and others [1-2]. In some cases of high noise pollution levels, intolerable reactions and negative impact become a threat to the well-being of humans and the environment [3]. Other studies contributed to the fact that noise pollution affects job performance and satisfaction, the health of humans and even animals [3-6]. Since there is a continuous population growth and increasing urbanization, noise pollution levels will automatically continue to increase; and this calls for comprehensive studies in this area of environmental pollution in order to be abreast of prevailing noise pollution levels and be able to predict future noise levels for proper planning of our cities and environmental management.

Predicting future noise pollution levels requires specialised customized modelling software and user know-how and as such make it difficult to obtain prediction models for a given location, especially in the developing world. Thus noise prediction has become a major challenge in urban planning and environmental management [7], where it is required to relate changes in spatial distribution of noise pollution levels for future urban expansion at the planning level and environmental management. Therefore mapping and forecasting of intraurban noise pollution change for urban development layout, still remains a very difficult task, since the formulation and application of the model depends on several factors, including the size of the area, availability of 
input data, which are largely land-use variables. Current efforts are mainly experiment-based, statistical models and noise mapping [7], and most literature focus on specific sources on noise such as transportation, industry, construction, and other social sources. Therefore all-inclusive models are required and efforts in this direction are largely dependent on land-use regression (LUR) modeling.

Land-use regression modelling approaches are mostly being used for assessing the exposure of the urban communities to air pollutions [8]. LUR methods use leastsquares regression modelling to predict air pollution levels based on predictor variables available. In the field of noise levels prediction, LUR modelling has been least explored. Currently, only two studies have applied this technique, the first one was applied in China and the other was applied in three European cities [7, 9]. In this present study, a generic LUR model was developed to predict noise pollution levels in the TMC using multiple linear regression (MLR). However, in order to improve on the modelling capabilities of the developed MLR approach in the noise prediction field, the analytical hierarchy process (AHP) was applied to formulate a hybrid model namely, AHP-MLR. This was done based on the strengths and the capabilities of the AHP, as in literature, to solve multi-criteria problems and also to rectify the inefficiencies associated with the application of MLR, since such study has not been comprehensively investigated [10-11]. Therefore, the aim of this present study is to compare and contrast the efforts of the two developed models (i.e. MLR and AHP-MLR) for noise levels prediction. This will help evaluate their performances, using statistical indicators, in noise prediction and to be able to select the most effective approach for predicting noise pollution levels in our communities, using the TMC as a case study.

\section{Materials and Methods Used}

\subsection{Study Area}

Tarkwa Mining Community (TMC) is a community found within the Tarkwa Nsuaem Municipality in the Western Region of Ghana. The study area is geographically located between latitudes $4^{\circ} 00^{\prime} 00^{\prime \prime} \mathrm{N}$ and $5^{\circ} 00^{\prime} 00^{\prime \prime} \mathrm{N}$ and longitudes $1^{\circ} 45^{\prime} 00^{\prime \prime} \mathrm{W}$ and $2^{\circ} 00^{\prime} 00^{\prime \prime} \mathrm{W}$. This community is about $89 \mathrm{~km}$ away from Takoradi, the Capital of the Western Region of Ghana [12].

The community is situated in an area well noted for the mining of minerals such as gold and manganese. Goldfields Ghana limited, Anglo-gold Ashanti, and Ghana Manganese Company are some of the large scale mining companies found in the TMC. There are also numerous allied mining companies located there. Several small scale mining activities are also going on in the TMC. Over the past few years TMC has seen infrastructural developments including road constructions, building of health posts, education, industries, banking, hospitality services and private business development [13]. Figure 1 shows a map of the Tarkwa Mining Community.

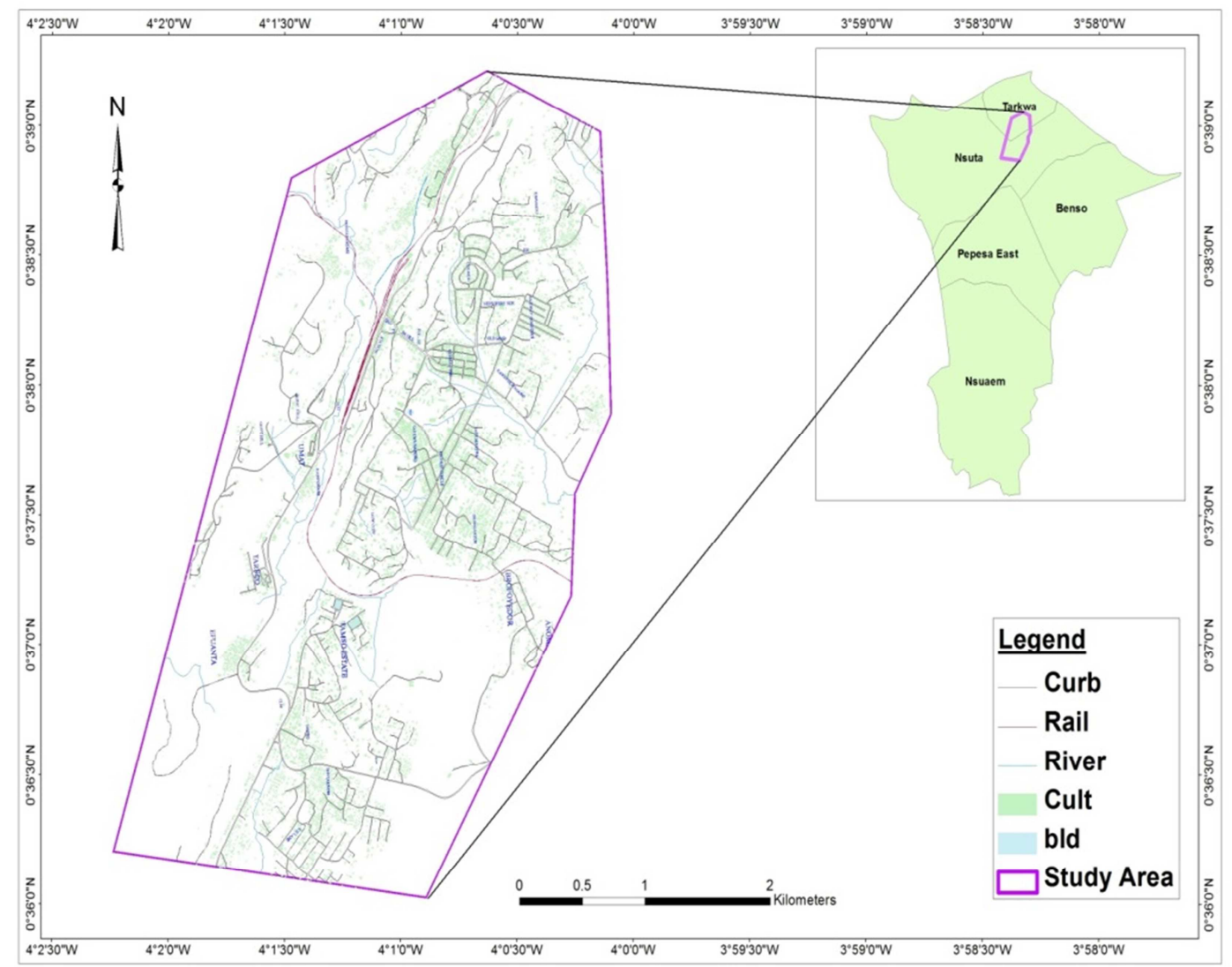

Figure 1. Tarkwa Mining Community. 


\subsection{Measurements of Noise Levels}

The spatial positions of the precise monitoring stations (PMS) in the TMC were measured using Garmin GPS 60CSx handheld Global Positioning System (GPS) of $2 \mathrm{~m}$ accuracy. A calibrated Larson Davis's SoundTrack LxT (Trade Mark) Sound Level Meter was also used to measure the noise levels in the TMC. The noise levels were measured from August 2014 to January 2015, which were outdoor nature. The measurements of the PMS were taken at street level and were also determined with the aid of the city digital map of the area.

The standard regulations for measuring noise levels were strictly adhered to. In order to avoid noise reflections, the instrument for measuring noise level was set on a tripod at about $1.5 \mathrm{~m}$ above the ground and also separated from the source by at least $1.5 \mathrm{~m}$. This decision was in consonance with what has been reported and accepted in the literature, including that of Mehdi and others who used $1.5 \mathrm{~m}$ above ground level and 1.22-1.52 $\mathrm{m}$ from the source of the noise [14]. The tolerance of the calibrated Larson Davis's Sound Track LxT trademark device is $\pm 0.6 \mathrm{dBA}$. A-weighted instantaneous sound pressure levels were recorded three times daily at the selected positions in the study area. The total number of the PMS used for the modeling was 50.

\subsection{Methods Used}

\subsubsection{Noise Prediction Models}

Land use regression models have been useful applications to predicting noise pollution levels in intraurban cities, but in this study the multiple linear regression (MLR) approach was applied. After developing the MLR model, a hybrid approach of AHP-MLR was also developed for forecasting the noise pollution levels in the TMC. In the afore-mentioned formulated models, the noise level was used as the dependent variable and the areas of the various land-uses within the study area were defined as independent variables.

The general equation therefore consisted of five independent variables namely land-use, traffic intensity, road network, distance to the main road, and population density. The AHP of the Multi-Criteria Decision Making (MCDM) was used to solve the complex multi-criteria decisions and develop the hybrid model. The equations developed from AHP were solved using matrix notations.

\subsubsection{The MLR Approach}

Equation (1) was used to develop the MLR model for the prediction of noise pollution levels in the TMC. The formulated observation equations were then solved using matrix notation. The general multiple linear regression expression for $k$ variables as given in Equation (1) as:

$$
y_{i}=\beta_{o}+\beta_{1} x_{i 1}+\beta_{2} x_{i 2}+\ldots+\beta_{k} x_{i k}+e_{i}, \quad i=1,2, \ldots, n
$$

In Equation (1), $y$ is the dependent variable which is the noise pollution levels in the TMC; $\beta_{1}, \beta_{2}$ and $\beta_{\mathrm{k}}$ are coefficients of the regression equation and $\beta_{0}$ is the intercept that is the value of $y$ when all $x$ s are zero.

Using the matrix notation a more compact framework was formed in terms of vectors representing the observations, levels of regressor variables, regression coefficients, and random errors. Therefore, Equation (1) was represented in a compact for in Equation (2) as:

$$
Y=X \beta+\varepsilon
$$

Where, $\mathrm{Y}$ is Noise Pollution levels, $\mathrm{X}$ represents the independent variables; $\epsilon$ is Residuals; and $A$ is the value of $Y$ when all $X$ s are zero. The least square estimator of the coefficients of the regression equation $(\beta)$ is given by the Equation (3):

$$
\beta=\left(X^{T} X\right)^{-1}\left(X^{T} Y\right)
$$

Since $\mathrm{X}$ is not usually a squared matrix, it is multiplied by the transpose of $\mathrm{X}$, that is $\mathrm{X}^{\mathrm{T}} \mathrm{X}$ and the inverse of $\left(\mathrm{X}^{\prime} \mathrm{X}\right)$ calculated. Hence the estimator $\beta$ calculated thus using Equation 3.

The predicted pollution noise levels were projected for ten years and were then used to develop the spatial distribution of the estimated noise levels.

\subsubsection{The Hybrid Approach (AHP-MLR)}

The AHP, one of the multi criteria analysis methodologies (MCDM) was selected for prediction model, a hybrid model formulated to improve the performance indicators of the single individual MLR model. This was done to rectify the inefficiencies associated with applying only MLR approach [10-11]. The AHP helps in making decisions using conflicting criteria of which each criterion has a particular level of importance in the final decision hence the need to quantify them. The AHP is a theory of comparative judgements through pairwise comparisons that relies on a comparison matrix at each level of the hierarchy. The comparisons are made using a scale of absolute judgements that represents how much more one element dominates another with respect to a given attribute [15].

One of the most crucial steps in many decision-making approaches is the accurate estimation of the pertinent data. This study involves pairwise comparison to create a ratio matrix. This was done by creating pairwise comparison inputs and producing relative weights as outputs. Thus applying decision-making attempt to determine the relative importance, or weight, of the alternatives in terms of each criterion involved in a given decision-making problem. The approach based on pairwise comparisons as proposed by Saaty has long attracted the interest of many researchers [16].

The levels of comparison are graduated on a scale of 1 to 9. The quantity 1normally represents two factors of same importance, whiles the quantity 9 represent a factor of extreme importance [16]. Based on literature as well as advice from experts' opinion, the judgements for the independent variables were formulated. Each independent variable has five alternatives and five decision criteria. Each 
of the alternatives was assessed in terms of the decision criteria and the relative importance (or weight) of each criterion. The results are thus represented in normalized pairwise comparison matrices in proceeding Equations 4 and 5.

$$
\bar{a}_{j k}=\frac{a_{j k}}{\sum_{l=1}^{m} a_{l k}}
$$

After creating the normalized pairwise comparison matrices for the independent variables, the criteria weight vector, w was built by averaging the entries on each row of $\mathrm{A}_{\text {norm }}$ that is,

$$
w_{j}=\frac{\sum_{l=1}^{m} \bar{a}_{j l}}{m}
$$

The whole processes were then summarised and then the judgment tables was represented by $5 \times 5$ matrices. After that they were then squared to obtain the eigenvector. The result was then normalised by summing the eigenvector and dividing each value of the eigenvector by the sum. The weights for the individual factors were obtained after the normalisation process. The process was then iterated a number of times until the weights assigned to each factor were consistent. A consistency ratio of 0.012 was achieved which is less than the maximum allowable ratio of 0.10 .

\subsubsection{Models Performance Evaluation}

To evaluate the accuracies of the models applied in this study, the under listed statistical indicators were calculated using the equations slated from Equation 6 to Equation 9. The equations are indicators helping to make unprejudiced evaluation of the models and they include Root Mean Square (RMSE), $\mathrm{R}^{2}$ which is coefficient of determination, $\mathrm{R}$ is correlation coefficient, mean and Standard Deviation.

$$
R M S E=\sqrt{\frac{\sum E^{2}}{N}}
$$

Where $\mathrm{n}$ is the number of observation points and $\mathrm{E}^{2}$ is the square of the error. The Mean Biased Error, MBE, was calculated using the formula in Equation 7:

$$
M B E=\frac{\sum E}{N}
$$

Where $\mathrm{E}$ is the error and $\mathrm{N}$ is the number of observation points. The MAE was calculated using the formula as in Equation 8:

$$
M A E=\frac{\sum|E|}{N}
$$

Where $|E|$ is the absolute error and $\mathrm{N}$ is the number of observation points. The SD was calculated using Equation 9:

$$
S D=\sqrt{\frac{\sum(x-\bar{x})^{2}}{N-1}}
$$

The Standard Deviation (SD) measures how closely the data are clustered around the mean, with $n-1$ being the degree of freedom.

\section{Results and Discussion}

\subsection{Results of the Errors in the Predictions}

\begin{tabular}{|c|c|c|c|c|}
\hline Observed & Predicted (AHP-MLR) & AHP-MLR (Error) & Predicted (MLR) & MLR (Error) \\
\hline 65 & 64.585 & 0.415 & 65.049 & -0.049 \\
\hline 78 & 78.335 & -0.335 & 77.741 & 0.259 \\
\hline 84 & 84.529 & -0.529 & 83.497 & 0.503 \\
\hline 84 & 83.639 & 0.361 & 82.54 & 1.46 \\
\hline 75 & 75.668 & -0.668 & 75.31 & -0.31 \\
\hline 86 & 85.204 & 0.796 & 84.635 & 1.365 \\
\hline 79 & 78.335 & 0.665 & 77.741 & 1.259 \\
\hline 85 & 84.927 & 0.073 & 83.821 & 1.179 \\
\hline 86 & 84.927 & 1.073 & 83.821 & 2.179 \\
\hline 89 & 91.755 & -2.755 & 90.995 & -1.995 \\
\hline 90 & 91.755 & -1.755 & 90.995 & -0.995 \\
\hline 91 & 93.059 & -2.059 & 92.183 & -1.183 \\
\hline 98 & 98.823 & -0.823 & 98.151 & -0.151 \\
\hline 96 & 93.801 & 2.199 & 92.887 & 3.113 \\
\hline 83 & 84.198 & -1.198 & 83.995 & -0.995 \\
\hline 81 & 82.894 & -1.894 & 82.807 & -1.807 \\
\hline 84 & 84.198 & -0.198 & 83.995 & 0.005 \\
\hline
\end{tabular}

Table 1 shows the errors propagated from the prediction of noise levels in the TMC using both prediction models of MLR and the hybrid, AHP-MLR, as compared with the observed.

Table 1. Errors Propagated During the Predictions of Noise Levels. 


\begin{tabular}{|c|c|c|c|c|}
\hline Observed & Predicted (AHP-MLR) & AHP-MLR (Error) & Predicted (MLR) & MLR (Error) \\
\hline 85 & 86.563 & -1.563 & 85.916 & -0.916 \\
\hline 75 & 72.883 & 2.117 & 74.28 & 0.72 \\
\hline 76 & 72.883 & 3.117 & 74.28 & 1.72 \\
\hline 74 & 72.883 & 1.117 & 74.28 & -0.28 \\
\hline 77 & 78.704 & -1.704 & 78.649 & -1.649 \\
\hline 79 & 78.704 & 0.296 & 78.649 & 0.351 \\
\hline 74 & 73.329 & 0.671 & 73.749 & 0.251 \\
\hline 73 & 73.329 & -0.329 & 73.749 & -0.749 \\
\hline 86 & 87.142 & -1.142 & 87.281 & -1.281 \\
\hline 88 & 90.678 & -2.678 & 89.6 & -1.6 \\
\hline 84 & 85.83 & -1.83 & 84.965 & -0.965 \\
\hline 89 & 92.254 & -3.254 & 91.951 & -2.951 \\
\hline 87 & 85.385 & 1.615 & 85.058 & 1.942 \\
\hline 89 & 92.239 & -3.239 & 91.328 & -2.328 \\
\hline 90 & 92.239 & -2.239 & 91.328 & -1.328 \\
\hline 95 & 96.977 & -1.977 & 95.626 & -0.626 \\
\hline 98 & 98.456 & -0.456 & 96.996 & 1.004 \\
\hline 97 & 96.977 & 0.023 & 95.626 & 1.374 \\
\hline 87 & 84.274 & 2.726 & 85.415 & 1.585 \\
\hline 86 & 84.274 & 1.726 & 85.415 & 0.585 \\
\hline 89 & 88.359 & 0.641 & 88.42 & 0.58 \\
\hline 93 & 93.733 & -0.733 & 93.321 & -0.321 \\
\hline 95 & 93.733 & 1.267 & 93.321 & 1.679 \\
\hline 94 & 92.254 & 1.746 & 91.951 & 2.049 \\
\hline 96 & 98.456 & -2.456 & 96.996 & -0.996 \\
\hline 92 & 92.239 & -0.239 & 91.328 & 0.672 \\
\hline 88 & 84.594 & 3.406 & 84.282 & 3.718 \\
\hline 80 & 78.704 & 1.296 & 78.649 & 1.351 \\
\hline 76 & 78.704 & -2.704 & 78.649 & -2.649 \\
\hline 68 & 71.835 & -3.835 & 71.756 & -3.756 \\
\hline
\end{tabular}

The results of the observed and the predicted noise levels are presented in Table 1. These are presented with locations of the PMS indicated along the major road (as explained earlier) and the computed values of noise level descriptors for the various locations in the TMC. Figure 2 shows the trend of errors as generated by both prediction models.

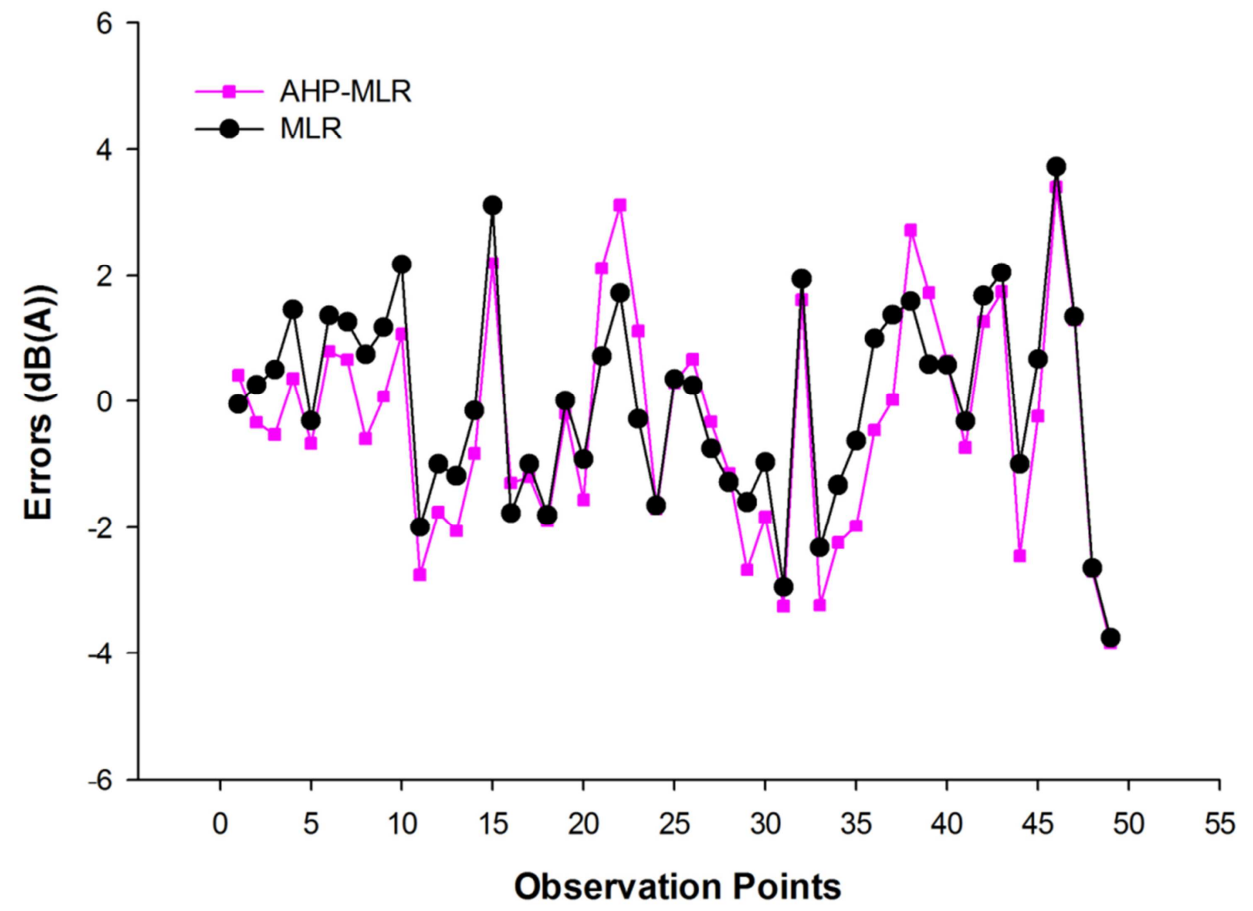

Figure 2. The Trend of Errors Generated from the Predictions Models.

The performances indicators of both models based on statistical analysis are presented in Table 2. The performance indicators include RMSE, Mean, Standard Deviation, $\mathrm{R}$ and $\mathrm{R}^{2}$, and these are compared for both the MLR and the AHP-MLR. 
Table 2. Comparison of the Two Prediction Models by Performance Indicators.

\begin{tabular}{|c|c|c|c|c|c|c|c|}
\hline \multirow{2}{*}{ Mathematical Model } & \multicolumn{7}{|c|}{ Performance Indicators } \\
\hline & RMSE & Mean & Standard Deviation & Maximum & Minimum & $\mathbf{R}^{2}$ & $\mathbf{R}$ \\
\hline AHP-MLR & 1.774 & 3.15 & 1.758 & 3.406 & -3.835 & 0.955 & 0.977 \\
\hline MLR & 1.569 & 2.462 & 1.585 & 3.718 & -3.756 & 0.961 & 0.98 \\
\hline
\end{tabular}

$\mathrm{RME}=$ root mean square error, $\mathrm{R}^{2}=$ coefficient of determination, $\mathrm{R}=$ correlation coefficient.

The performance evaluation comparison is further illustrated in Figure 3 using the box-and-whisker plots. The box-and-whisker plot presented is an exploratory graphic showing the spatial distribution of the errors. These are used in this study to illustrate the distribution of error propagation

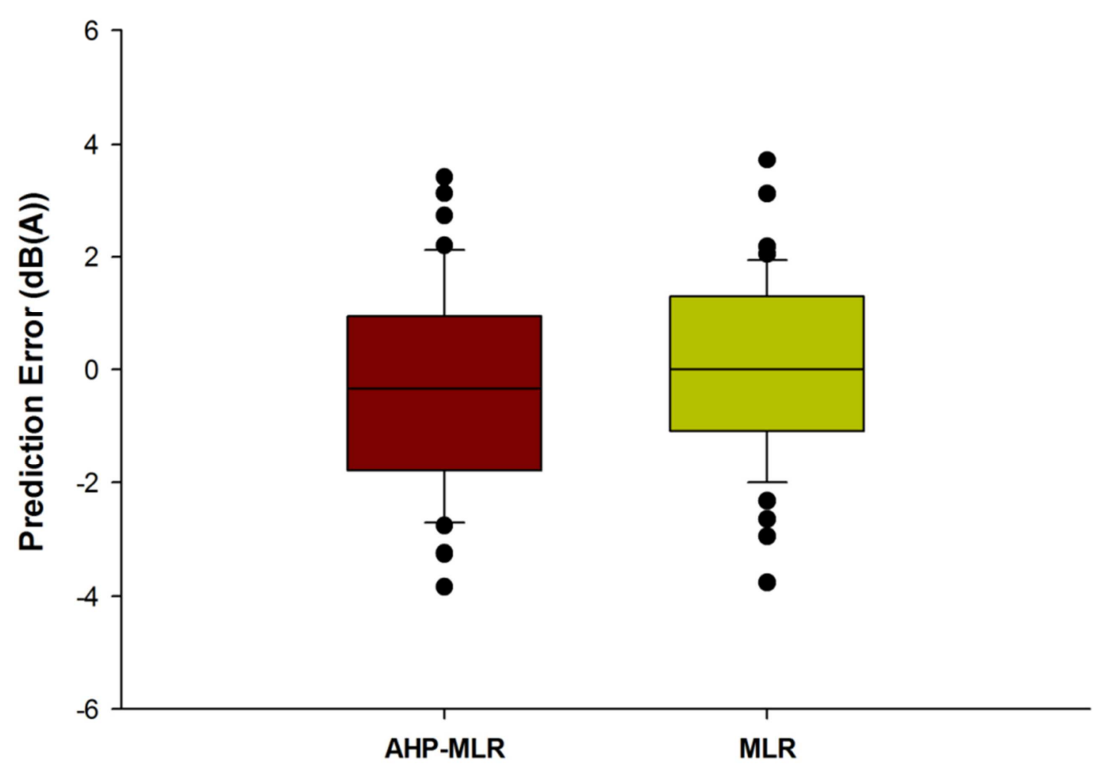

Figure 3. Error Variability of the MLR and AHP-MLR.

The evaluation of the performances of the designed and implemented models was also presented in range plots (Figure 4) for further analysis. Range plots also illustrated the minimum, maximum and average errors propagated when both MLR and AHP-MLR approaches were applied to predicting noise pollution levels for the TMC. The range plot gave further confirmation of the propagated errors as shown in Figure 4, showing the minimum, mean and maximum errors of both models.

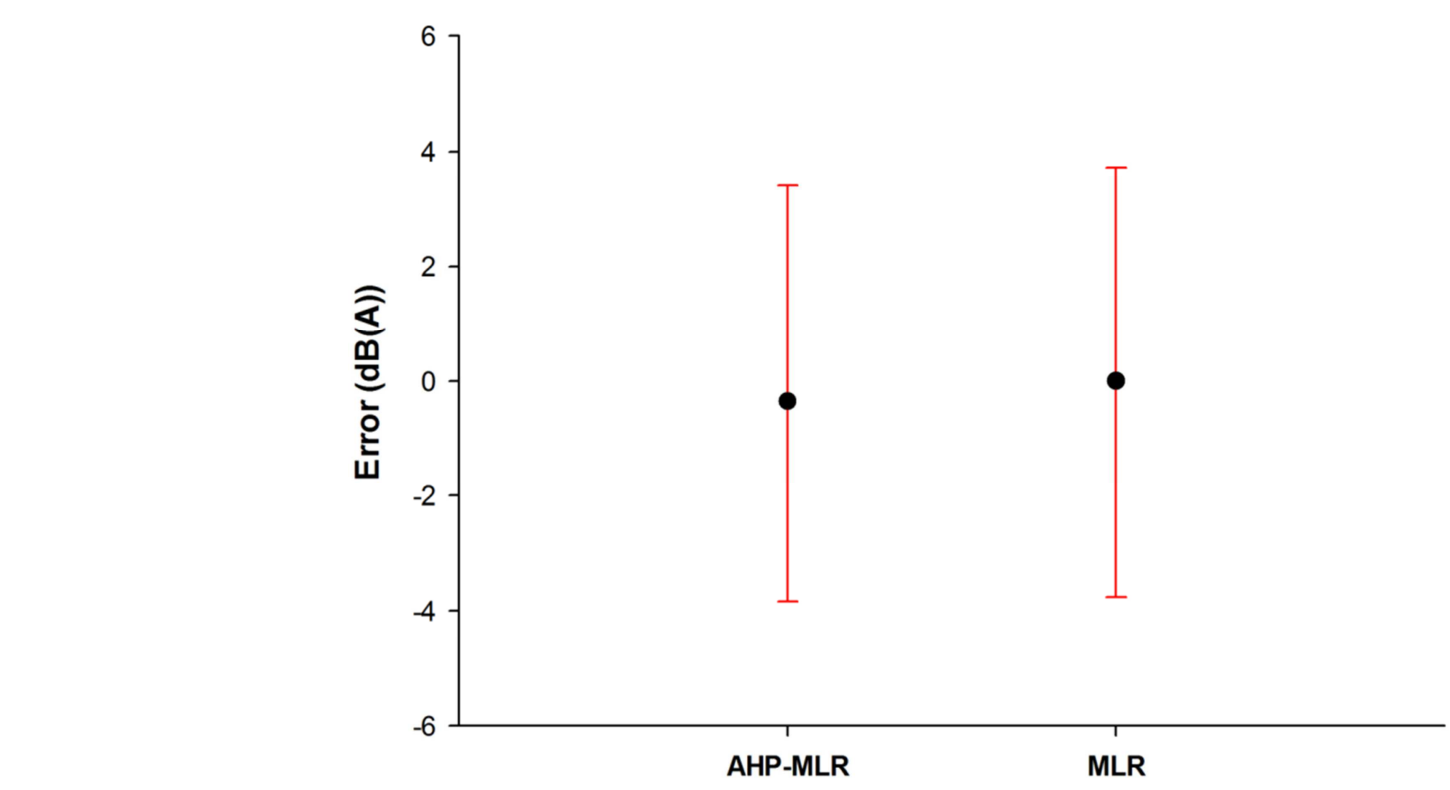

Figure 4. Error Bar for both MLR and AHP-MLR Models. at a glance. The comparison in this case stem from outliers, through lower and upper whiskers, lower and upper quartiles, and the median. The box-and-whisker of course shows more than just four split groups. It can also be seen which way the data sways by comparing both models. 
The results from the developed models were used to plot the spatial distribution of the noise levels of the study area. The spatial distribution of the estimated noise pollution levels from the monitoring stations in the TMC forecasted are presented in Figure 5 demonstrating that GIS could be used for noise mapping.

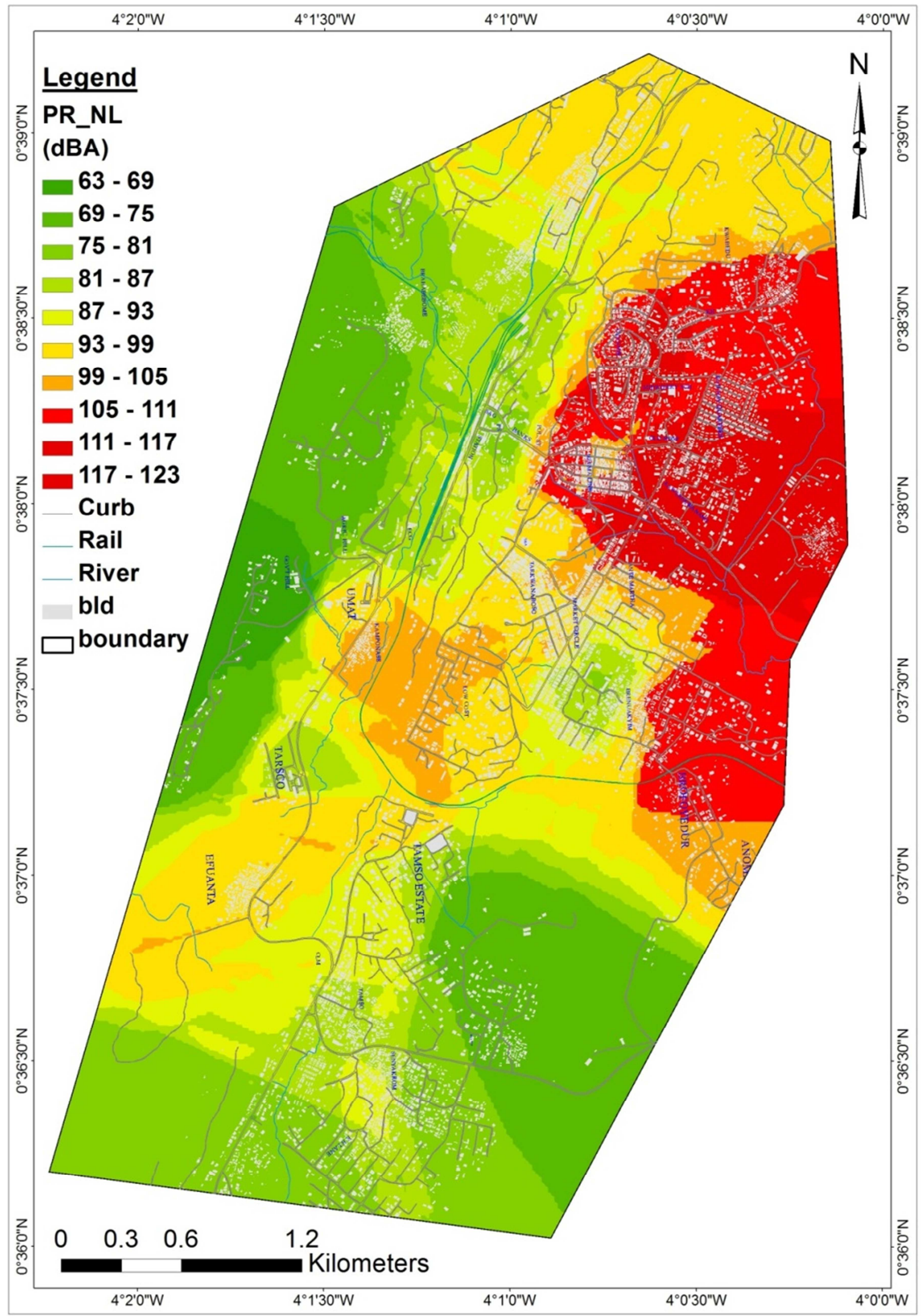

Figure 5. Noise Map for the Predicted Noise Levels. 


\subsection{Discussion}

The residuals indicating the degree at which the predicted outputs produced by the MLR and AHP-MLR models depart from the corresponding measured noise data are presented in Table 1. These residuals also suggest the prediction inadequacies of the methods utilized in this study. This will further give the modeler and the user the opportunity to know evidently the quantitative predictive strength of the two methods. This assertion is clearly demonstrated from the analysis of Table 1 and Figure 2 where it was shown that the MLR technique produced more satisfactory results than the AHP-MLR. The inference made here is that the MLR was able to model appropriately on the measured noise data as compared with the AHP-MLR. In the light of these, it could be stated that the predicted outputs rendered by the MLR are in better agreement to the measured noise level data than the AHP-MLR. These assertions are further confirmed by Figure 2, where it can be observed that the extent of error variability for the MLR appears to be better across the zero value than the AHP-MLR, respectively.

It is well established in literature that to determine the extent of fit of a model, the RMSE is a good estimator. From Table 2, it is a noticed that the MLR produced better performance in relations to the statistical findings. Thus, the closer the RMSE value to zero the better the model prediction strength. On the basis of the RMSE results, it can be observed that the MLR outperformed the AHP-MLR. Moreover, the $\mathrm{R}^{2}$ and $\mathrm{R}$ values (Table 2) produced further affirmed the quality of the prediction performance of the two methods. Here, $\mathrm{R}^{2}$ values obtained indicate the level of tolerance of the prediction values from MLR and AHP-MLR. Thus, 96.1\% changes in the measured noise level data are explained by the variation in the MLR predicted output values while, the AHP-MLR could explain only $95.5 \%$ variability. The $\mathrm{R}$ findings on the other hand show a degree at which the predicted noise data fall closely to the line of best fit. Judging from the R outcomes in Table 2, the MLR model delineates discrepancy in the predicted data with high precision and accuracy. This precision capability of the models can also be seen from the standard deviation values (Table 2).

The analysis performed on the two models by comparing the box-and-whisker plots (Figure 3) helps to understand the spread of error distribution with respect to the predicted noise level data. On each plot, the central mark is the error median, the edges of the box is the first and third quartile, and the lower and upper whiskers signify the minimum and maximum error range not considered as outliers. The essence of the box-whisker plot (Figure 3) is to provide a graphical rendition of the summary statistics based on the residuals achieved by the MLR and AHP-MLR. With reference to Figure 3, it can be seen that the MLR achieved less error variability than AHP-MLR model. It is also evident from Figure 3 where it can be noticed that the interquartile range length for the MLR is smaller than the AHP-MLR.
Furthermore, the range plot (Figure 4) gave further confirmation of the previous assessment of the performance indicators. The range plot showed the minimum (lower whisker), mean (middle) and maximum (upper whisker) errors for both developed models (Figure 4). Due the strength of the MLR model developed, it was used to forecast longterm variability of urban noise levels in the TMC. Additionally, the noise map developed from the forecasted noise levels brought to bear the ample use of GIS for noise mapping. The results obtained, as shown in Figure 5, indicate that it is possible to develop a LUR model using the MLR technique with independent variables and that the results could be used for noise mapping (Figure 5).

It is more important to note that the noise prediction models developed in this research are exclusively different from the already existing LUR models developed by [7, 9]. In these current applications the variety of independent variables both of the MLR and AHP-MLR equations are entirely different from the previous LUR models developed. It is also different from other basic prediction models by the consideration of land-use and other relevant variables. The results from the modeling processes show that with accurate data, noise prediction models are now promising tools, as demonstrated, for noise exposure assessment with potential applications in urban planning, environmental management particularly in areas where noise predictions models or noise maps from competent authorities are not available.

\section{Conclusion}

Two unique noise prediction models have been developed and their performances evaluated, from MLR and then AHPMLR methods, using statistical indicators. Based on the strengths and the capabilities of AHP, a hybrid model of AHP-MLR was design to augment the inefficiencies of MLR and improve on it. However, comparing the performances of both models using statistical indicators, MLR rather performed better than the hybrid AHP-MLR. The difference reflected in the indicators whereby RMSE of MLR is 1.569 and that of AHP-MLR is 1.774. Moreover, $\mathrm{R}^{2}$ and $\mathrm{R}$ of MLR are 0.961 and 0.980 respectively whiles those of AHP-MLR are 0.955 and 0.977 respectively. Furthermore, visual inspection of the box-and-whisker plot cum that of the range plot further confirms the performances of the two developed models.

In such situation, to be able to set up a standard practice of noise prediction especially in Ghana, the hybrid approach of AHP-MLR and MLR are proposed over the traditional models. This will further accelerate effective and accurate prediction models for noise predictions. The spatial distribution of the long-term predicted noise levels demonstrates the values of these models to mapping urban noise levels in relation with urban land use change at the same time being feasible for different applications. The application of land-use variables also shows that the developed models can be easily applied to predict noise 
pollution levels and also identify potential areas that are violating the regulatory requirements.

In conclusion, the developed models have also confirmed its ability in mapping intraurban noise in relation to urban land-use as changes occur. This rather will very much aid in urban planning and environmental noise management. It could also be applied predict environmental noise changes with time and epidemiological studies as well as decisionmaking tool. It was observed from this study that the more the positional monitoring stations observed, the better the model performance. Therefore, the model performs better for large scale area like the study area and vice versa.

\section{References}

[1] Ylikoki J, Juntunen J, Matiainen E., Ylikoski M, Ojala M. (1988). Sub clinical Vestibular pathology $\mathrm{n}$ patients with noseinduced hearing loss from intense impulse noise. Acta Otolaryngology, 105: pp.588 -563.

[2] Satterfield K. (2001). Balance disorders and patients with NIHL in on ear. America Academy of Otolaryngology Head and Neck Surgery, Vol. 703, pp. 1-3.

[3] Goines, L. and Hagler, L. (2007), "Noise Pollution: A Modern Plaque", Southern Medical Journal, Vol. 100, Pp. 287-294.

[4] Benfield, J. A., Nurse, G. A., Jakubowski, R., Gibson, A. W., Taff, B. D., Newman, P. and Bell, P. A. (2012), "Testing Noise in the Field: A Brief Measure of Individual Noise Sensitivity", Environment and Behavior, Vol. 46, No. 3, pp. 353-372.

[5] Ighoroje, A. D. A, Marchie, C., and Nwobodo, E. D. (2004), Noise-Induced Hearing Impairment as an Occupational Risk Factor among Nigerian Traders, Nigerian Journal of Physiological Sciences 19 (1-2): 14-19, Physiological Society of Nigeria 2004.

[6] Passchier-Vermeer, W., and Passchier, W. F., (2000), Noise Exposure and Public Health, Environmental Health Perspective, Vol. 108, p. 123-131.

[7] Xie, D., Liu, Y., and Chen, J., (2011), "Mapping Urban Environmental Noise: A Land Use Regression Method", Environmental, Science and Technology, Vol. 45, 7358-7364.
[8] Henderson, F., Cox, F., Ganesh, S., Jonker, W., Young, W. (2015), "Rumen Microbial Community Composition Varies with Diet and Host, but a Core Microbiome Is Found Across A Wide Geographical Range", Journal of Expo Science Environment.

[9] Aguilera, I., Foraster, M., Basagaña, X., Corradi, E., Deltell5, A., Morelli, X., Phuleria, H. C., Ragettli, M. S., Rivera, M., Thomasson, A., Rémy Slama, R., and Künzli, N., (2015), "Application of Land-use Regression Modelling to Assess the Spatial Distribution of Road Traffic Noise in Three European Cities.”, Journal of Expo Science Environ Epidemiol. Vol. 25 (1): 97-105.

[10] Akinlalu, A., A., Adegbuyiro, A., Adiat, K., A., N., Akeredolu, B. E., and Leteef, B. E. (2017), "Application of multi-criteria decision analysis in prediction of groundwater resources potential: A case of Oke-Ana, Ilesa Area Southwestern, Nigeria.", NRIAG, Journal of Astronomy and Geophysics, Vol. 6, Issue 1, June 2017, pp. 184-200.

[11] Xishang, D., Huaizhi, M., and Zhen, Y. (2014), "An Application of AHP and FAHP on the Model Prediction", Applied Mechanic and Materials, Vols. 687-691, pp. 16411644.

[12] Mantey, S. and Tagoe, N. D. (2012) "Geo-Property Tax Information System - A Case Study of the Tarkwa Nsuaem Municipality, Ghana”, FIG Working Week 2012: Knowing to manage the territory, protect the environment, evaluate the cultural heritage", Rome Italy, from 6th to 10th, May 2012.

[13] Kumi-Boateng, B. (2012), “A Spatio-Temporal Based Estimation of Land-Use Cover Change and Sequestered Carbon in the Tarkwa Mining Area of Ghana", PhD Thesis, University of Mine and Technology, Tarkwa, 2012, pp. 163.

[14] Mehdi, M. R., Minho, K., Jeong, C. S. and Mudassar, H. A. (2010), "Spatio-Temporal Patterns of Road Traffic Noise Pollution in Karachi, Pakistan", Environment International journal, Vol. 12, pp. 1-8.

[15] Saaty, T. L. (1980), The Analytical Hierarchy Process, McGraw-Hill, pp. 23-157.

[16] Xu, W. and Yang, N. (2014),"Lithium Metal Anodes for Rechargeable Batteries", Energy Environ. Sci. Vol. 7, pp. 513-537. 